\title{
Insulin Glargine Combined with Oral Antidiabetic Drugs for Asians with Type 2 Diabetes Mellitus: A Pooled Analysis to Identify Predictors of Dose and Treatment Response
}

\author{
Tianwei Gu • Ting Hong • Pengzi Zhang • Sunyinyan Tang • \\ Yan Bi · Hai Lu · Lichuang Men · Dongwei Ma · Dalong Zhu
}

Received: December 4, 2017 / Published online: March 9, 2018

(C) The Author(s) 2018. This article is an open access publication

\begin{abstract}
Introduction: In Asia, patients with type 2 diabetes mellitus (T2DM) often have suboptimal glycemic control for many years prior to initiating basal insulin. Active titration of basal insulin is also required to improve glycemic outcomes. This pooled analysis was conducted to determine the impact of patient baseline covariates on the required dose of basal insulin and treatment response, for the improved management of Asian patients with T2DM.

Methods: Data on insulin-naïve Asian patients with T2DM who initiated and fully titrated insulin glargine $100 \mathrm{U} / \mathrm{mL}$ (Gla-100) for
\end{abstract}

Tianwei Gu and Ting Hong are co-first authors.

Enhanced content To view enhanced content for this article go to https://doi.org/10.6084/m9.figshare. 5868147.

Electronic supplementary material The online version of this article (https://doi.org/10.1007/s13300018-0381-9) contains supplementary material, which is available to authorized users.

T. Gu $\cdot$ T. Hong $\cdot$ P. Zhang $\cdot$ S. Tang $\cdot$ Y. Bi ·

D. Zhu ( $\triangle)$

Department of Endocrinology, Drum Tower

Hospital Affiliated to Nanjing University Medical

School, Nanjing, China

e-mail: zhudalong@nju.edu.cn

H. Lu · L. Men · D. Ma

Sanofi Investment Co., Ltd., Shanghai, China $\geq 20$ weeks were pooled from seven randomized, controlled, treat-to-target trials. Covariance and multivariate linear/logistic regression analyses were applied to determine the impact of the baseline covariates on Gla-100 dose (primary outcome) and treatment response (secondary outcomes) at week 24 for patients from Asia $(N=724)$ and from China alone $(n=249)$. Based on the multivariate analysis for the primary outcome in the Asian population, a nomogram was developed.

Results: The dose of Gla-100 at week 24 was negatively correlated with age and positively correlated with body mass index (BMI) and fasting plasma glucose (FPG) at baseline in both Asian and Chinese populations. In both populations, higher baseline glycated hemoglobin $\left(\mathrm{HbA}_{1 \mathrm{c}}\right)$ was associated with a lower reduction in $\mathrm{HbA}_{1 \mathrm{c}}$ from baseline, higher $\mathrm{HbA}_{1 \mathrm{c}}$ at week 24, and a lower chance of achieving $\mathrm{HbA}_{1 \mathrm{c}}<7 \%$ at week 24 . The constructed nomogram enables calculation of the likely dose of Gla-100 required by Asian patients with T2DM to achieve $\mathrm{HbA}_{1 \mathrm{c}}<7 \%$ at week 24 .

Conclusions: Higher doses of Gla-100 are likely to be required in younger patients or patients with higher baseline BMI or FPG. The nomogram developed in this study can aid clinicians to titrate the dose of Gla-100 appropriately. Evidence in this pooled analysis also indicates that initiating basal insulin at a lower $\mathrm{HbA}_{1 \mathrm{c}}$ can lead to greater glycemic control.

Funding: Sanofi China Investment Company. 
Keywords: Asia; Basal insulin; China; Dose titration; Insulin glargine $100 \mathrm{U} / \mathrm{mL}$; Nomogram; Pooled analysis; Type 2 diabetes mellitus

\section{INTRODUCTION}

Type 2 diabetes mellitus (T2DM) is a progressive disease often requiring add-on therapy, as many patients remain at suboptimal glycemic control on oral antidiabetic drugs (OADs) alone $[1,2]$. The addition of basal insulin is recommended by guidelines as one of the initial treatment intensification options in these patients [3-6]. Despite recommendations for timely initiation, data indicate that Asian patients with T2DM have suboptimal glycemic control for approximately 6-9 years, with average glycated hemoglobin $\left(\mathrm{HbA}_{1 \mathrm{c}}\right)$ levels of $9.2-10.5 \%$ at the point of basal insulin initiation [7-9]. In Asian patients, delays in basal insulin initiation are associated with a lower reduction in $\mathrm{HbA}_{1 \mathrm{c}}$, as well as a lower proportion of patients being able to achieve $\mathrm{HbA}_{1 \mathrm{c}}<7 \%$ [7].

In addition to the issues surrounding timely initiation of basal insulin in Asia, there are several challenges pertaining to the titration of basal insulin. In clinical practice, Asian patients with T2DM who have a lower body mass index (BMI) than their Caucasian counterparts [10-12] are often perceived to be at an increased risk of hypoglycemia, which leads to conservative treatment goals and a cautious approach to dose titration by physicians $[9,13]$. Large, realworld studies conducted in Asia, such as The First Basal Insulin Evaluation (FINE) Asia registry study and the Observational Registry of Basal Insulin Treatment (ORBIT) study, have hypothesized that further active titration of basal insulin can potentially increase the proportion of patients with T2DM achieving adequate glycemic control and improve glycemic outcomes [9, 14].

There is a growing body of evidence describing the importance of individualizing the management of T2DM based on ethnicity [15-17]. Therefore, when titrating the dose of basal insulin, it is important to consider the ethnic and genetic differences between Asians and Caucasians [18, 19], which may lead to different insulin requirements in both of these populations [20]. Understanding the specific insulin needs of Asian populations requires greater understanding of the patient-level factors that impact their required dose of basal insulin and treatment response.

The current study is a pooled analysis of Asian patients included in treat-to-target (TTT) randomized controlled trials (RCTs) of insulin glargine $100 \mathrm{U} / \mathrm{mL}$ (Gla-100). It was designed to investigate the relationship between patient baseline covariates, basal insulin dose, and treatment outcomes in insulin-naïve Asian patients with T2DM who have suboptimal glycemic control with OADs. To further explore the relationship between patient characteristics and outcomes, separate analyses were performed for the main population of patients from Asia and for a subpopulation of patients from China. The aim was not to compare both populations, but rather to lay the foundation for future analyses specific to the Chinese population and provide information to guide local clinical practice.

\section{METHODS}

\section{Study Design and Population}

This was a pooled analysis of Asian patients included in seven TTT RCTs [1, 21-27]. Details of the seven RCTs, including their respective titration algorithms, are provided in Table 1. Trials were identified on the basis of a search of the study sponsor's internal database for all TTT RCTs that it has conducted involving treatment with Gla-100 for at least 24 weeks in patients with T2DM uncontrolled with OADs. A total of 14 trials were identified; however, seven were excluded on the basis of the small number of patients in each trial fulfilling the inclusion criteria. Inclusion criteria were any insulinnaïve Asian patient in the full analysis set (FAS) population of the individual trials aged $>18$ years with T2DM previously uncontrolled with OADs, with $\mathrm{HbA}_{1 \mathrm{c}} \geq 7.0 \%$ and $\leq 12.0 \%$, who had initiated and fully titrated Gla-100 according to a TTT algorithm for at least 20 weeks during the course of the individual 


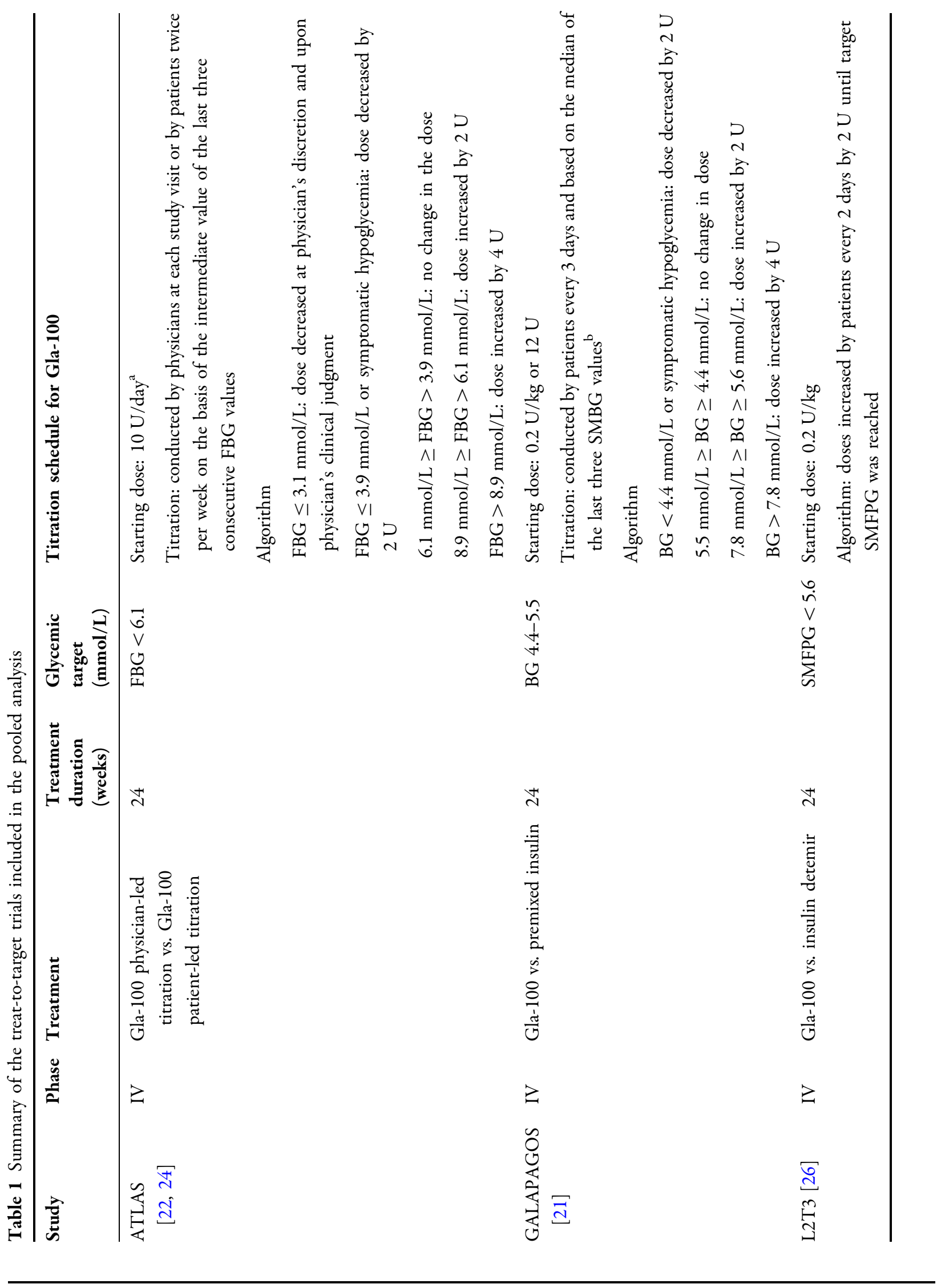




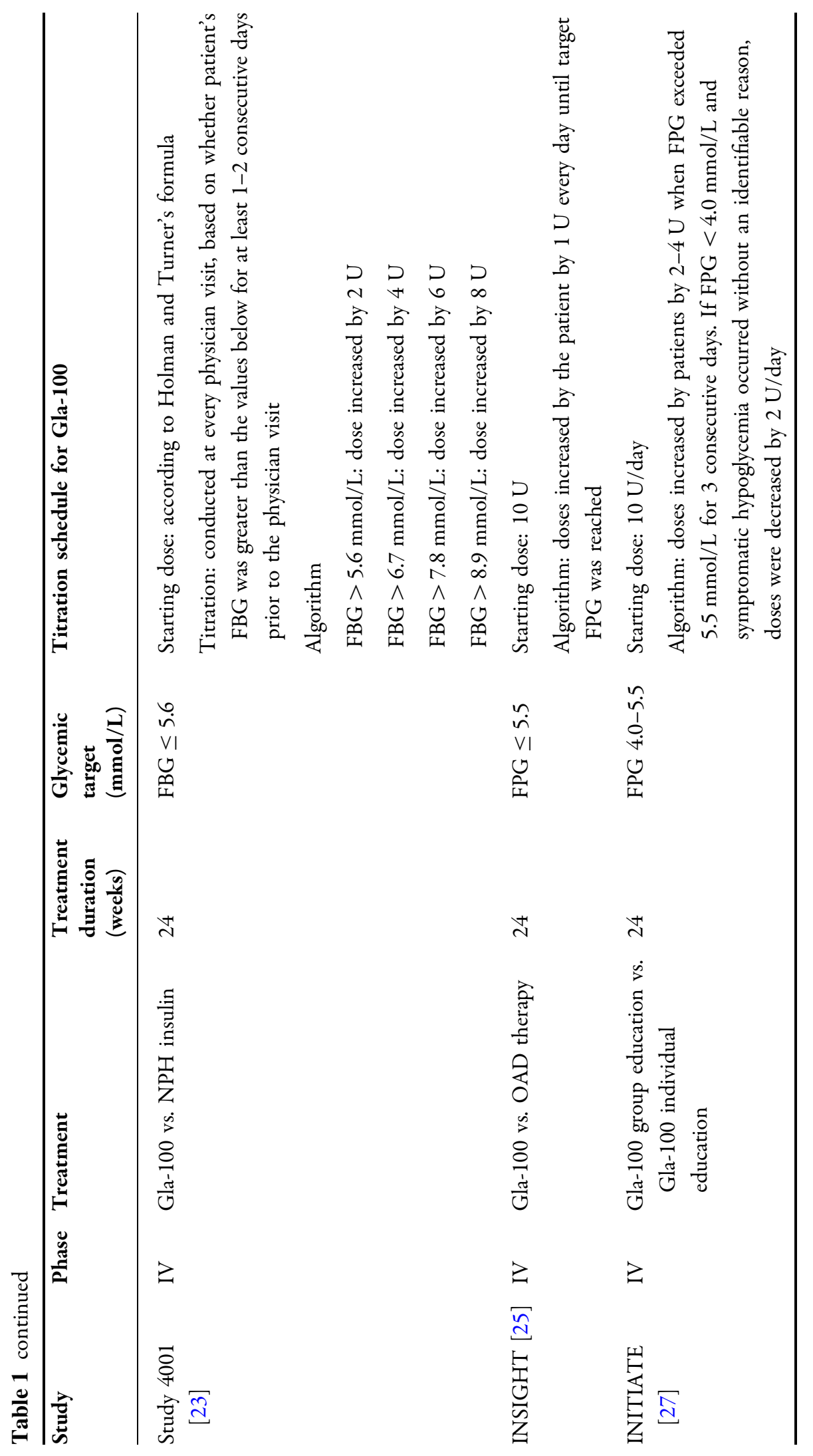




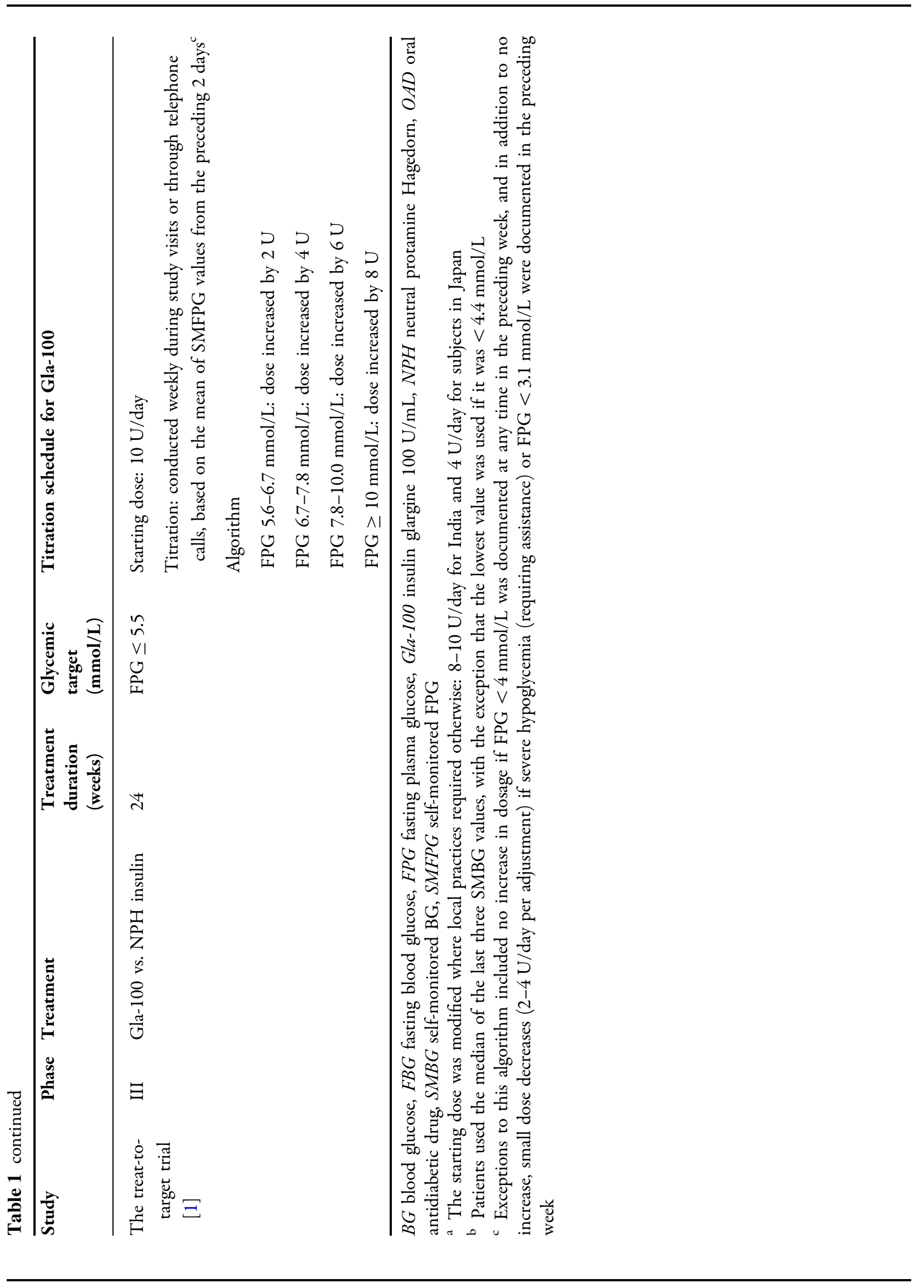


RCTs. Patients were excluded in the individual trials if they had diabetes other than T2DM, acute diabetic complications (including unexplained severe hypoglycemia in the last 6 months), clinically significant acute major organ or systemic disease, or if they were pregnant or lactating. As this was a pooled analysis of pre-existing data, no ethical approval was required to conduct the study. This article is based on previously conducted studies and does not involve any new studies of human or animal subjects performed by any of the authors.

\section{Study Outcomes}

The primary outcome of this pooled analysis was identification of the baseline predictors of the dose of Gla-100 at week 24 in both the Asian population and in the subpopulation of patients from China. Based on the identified baseline predictors in the Asian population, a nomogram was developed for numerical determination of the dose of Gla-100 likely to be required to achieve target glycemic control $\left(\mathrm{HbA}_{1 \mathrm{c}}<7 \%\right)$ at week 24 . Secondary outcomes were identification of the baseline predictors for treatment response and the dose of Gla-100 in units per kilogram per day at week 24 in both study populations. The different measures of treatment response were $\mathrm{HbA}_{1 \mathrm{c}}$ value at week 24, achievement of target glycemic control $\left(\mathrm{HbA}_{1 \mathrm{c}}<7 \%\right)$ at week 24, $\mathrm{HbA}_{1 \mathrm{c}}$ response (calculated as $\mathrm{HbA}_{1 \mathrm{c}}$ at week 24 - baseline $\mathrm{HbA}_{1 \mathrm{c}}$ ), and fasting plasma glucose (FPG) level at week 24 .

\section{Statistical and Analytical Procedures}

Eligible patients who were in the FAS population of the seven individual RCTs comprised the analysis population of this study. The baseline covariates explored were age, sex, weight, BMI, duration of diabetes, FPG, postprandial plasma glucose (PPG), $\mathrm{HbA}_{1 \mathrm{c}}$, and number of OADs prescribed. Correlation analyses were performed among the baseline covariates for the primary and secondary outcomes using analysis of covariance (ANCOVA). Spearman correlation coefficients and their associated $P$ values were computed for each pairwise correlation.
Following the covariance analyses, multivariate analyses were conducted. Forward selection of the baseline covariates from the covariance to the multivariate models was performed on the basis of an entry selection criterion of $\alpha=0.2$. A multivariate, generalized linear regression model was run with all the baseline covariates that satisfied the forward model selection criterion. Parameter estimates and their associated 95\% confidence intervals (CI) and $P$ values were calculated for the selected baseline covariates included in the multivariate model.

For achievement of target glycemic control at week 24, a logistic regression model was used for the covariance analysis, and a multivariate logistic regression model was run with all the baseline covariates that satisfied the entry selection criterion, in accordance with the other analyses.

The nomogram was constructed on the basis of results of the multivariate analysis of the primary outcome in the FAS Asian population. In developing the nomogram, the linear predictor method was used to assign points to characteristics and predictions from the multivariate model to map cumulative point totals. The nomogram is a representation of the results of the multivariate analysis as a whole and includes all of the patient baseline covariates within the analysis, regardless of whether they were significant or not.

All analyses were conducted separately in the FAS Asian population and subpopulation of patients from China. Data were analyzed using SAS 9.2 (Cary, NC, USA) or a later version, and the nomogram was plotted using SAS 9.4 (Cary, NC, USA). Variables with $P<0.05$ in the covariance or multivariate analyses were considered as significant predictors of the primary and secondary outcomes.

\section{RESULTS}

\section{Patients}

\section{Participant Selection}

A total of 724 Asian patients from the seven TTT RCTs satisfied the study eligibility criteria and 
Table 2 Baseline characteristics of the study population

\begin{tabular}{lll}
\hline Category & $\begin{array}{l}\text { All patients } \\
(N=724)\end{array}$ & $\begin{array}{l}\text { Chinese patients } \\
(n=249)\end{array}$ \\
\hline
\end{tabular}

Age, years

$\begin{array}{lll}N & 723 & 248 \\ \text { Mean } & 55.9(8.79) & 57.7(8.21) \\ (\text { SD) } & & \end{array}$

Sex, $n(\%)$

$\begin{array}{lll}\text { Female } & 311(43.0) & 117(47.0) \\ \text { Male } & 412(56.9) & 131(52.6) \\ \text { Missing } & 1(0.1) & 1(0.4) \\ \text { Race, } n(\%) & \\ \text { Asian/ } & 724(100.0) & 249(100.0) \\ \text { oriental } & \\ \text { Country, } n(\%) & \\ \text { China } & 249(34.4) & 249(100.0) \\ \text { Others } & 475(65.6) & 0\end{array}$

Weight, kg

$\begin{array}{lll}N & 724 & 249 \\ \text { Mean } & 70.2(12.41) & 69.4(11.03) \\ (\mathrm{SD}) & & \end{array}$

BMI, $\mathrm{kg} / \mathrm{m}^{2}$

$\begin{array}{lll}N & 724 & 249 \\ \text { Mean } & 26.4(4.02) & 25.5(3.36) \\ (\mathrm{SD}) & & \end{array}$

Duration of diabetes, years ${ }^{\mathrm{a}}$

$\begin{array}{lll}N & 711 & 249 \\ \text { Mean } & 9.7(6.28) & 9.6(5.78) \\ (\mathrm{SD}) & \end{array}$

FPG, $\mathrm{mmol} / \mathrm{L}$

$\begin{array}{lll}N & 718 & 249 \\ \text { Mean } & 9.0(2.30) & 8.9(2.08) \\ (\mathrm{SD}) & & \end{array}$

PPG, mmol/L

\begin{tabular}{lll}
$N$ & 691 & 245 \\
\hline
\end{tabular}

Table 2 continued

\begin{tabular}{lll}
\hline Category & $\begin{array}{l}\text { All patients } \\
(\boldsymbol{N}=724)\end{array}$ & $\begin{array}{l}\text { Chinese patients } \\
(\boldsymbol{n}=\mathbf{2 4 9})\end{array}$ \\
\hline $\begin{array}{c}\text { Mean } \\
(\mathrm{SD})\end{array}$ & $12.8(3.67)$ & $12.4(2.91)$ \\
$\mathrm{HbA}_{1 \mathrm{c}}, \%$ & & \\
$N$ & 724 & 249 \\
$\begin{array}{c}\text { Mean } \\
(\mathrm{SD})\end{array}$ & $8.7(1.03)$ & $8.5(1.05)$
\end{tabular}

Number of OADs

\begin{tabular}{lll}
0 & $4(0.6 \%)$ & 0 \\
1 & $66(9.1 \%)$ & $41(16.5 \%)$ \\
2 & $599(82.7 \%)$ & $208(83.5 \%)$ \\
3 & $54(7.5 \%)$ & 0 \\
4 & $1(0.1 \%)$ & 0 \\
\hline
\end{tabular}

$B M I$ body mass index, $F P G$ fasting plasma glucose, $H b A_{1 c}$ glycated hemoglobin, $O A D s$ oral antidiabetic drugs, $P P G$ postprandial glucose, $S D$ standard deviation

${ }^{a}$ Duration of diabetes $=$ informed consent form signed date - diabetes start date. If the diabetes start date was partial or missing, 6 was used to impute the month and 15 was used to impute the day

were included in this pooled analysis. The majority of patients (including the subgroup of 249 patients from China) were from the ATLAS [22, 24] and GALAPAGOS [21] studies. Details of the studies are provided in Table 1 and patient disposition is shown in Table S1 in the supplementary material.

\section{Baseline Characteristics}

The baseline characteristics of included patients are summarized in Table 2. Overall, $34.4 \%$ of patients were from China and the remainder were from other Asian countries. In the Asian population, mean age was $55.9 \pm 8.79$ years, $56.9 \%$ were male, and $82.7 \%$ were receiving two OADs at the time of the study. In the Chinese subpopulation, mean age was $57.7 \pm 8.21$ years, $52.6 \%$ were male, and $83.5 \%$ were receiving two OADs. 
Table 3 Patient data at week 24

\begin{tabular}{|c|c|c|}
\hline Category & All patients $(N=724)$ & Chinese patients $(n=249)$ \\
\hline \multicolumn{3}{|l|}{ FPG, $\mathrm{mmol} / \mathrm{L}$} \\
\hline Mean (SD) & $6.1(1.52)$ & $6.0(1.23)$ \\
\hline \multicolumn{3}{|l|}{ Gla-100 dose, U } \\
\hline Mean (SD) & $25.7(17.31)$ & $22.4(11.20)$ \\
\hline \multicolumn{3}{|l|}{ Gla-100 dose, U/kg/day } \\
\hline Mean $(S D)$ & $0.4(0.23)$ & $0.3(0.15)$ \\
\hline \multicolumn{3}{|l|}{$\mathrm{HbA}_{1 \mathrm{c}}, \%$} \\
\hline Mean (SD) & $7.5(0.96)$ & $7.2(0.82)$ \\
\hline \multicolumn{3}{|l|}{$\mathrm{HbA}_{1 \mathrm{c}}$ response, $\%$} \\
\hline Mean (SD) & $-1.3(1.10)$ & $-1.3(1.07)$ \\
\hline \multicolumn{3}{|c|}{ Achieving target glycemic control } \\
\hline $\mathrm{HbA}_{1 \mathrm{c}}<7 \%, n(\%)$ & $231(31.9 \%)$ & $103(41.4 \%)$ \\
\hline $\mathrm{HbA}_{1 \mathrm{c}} \geq 7 \%, n(\%)$ & $493(68.1 \%)$ & $146(58.6 \%)$ \\
\hline
\end{tabular}

FPG fasting plasma glucose, Gla-100 insulin glargine $100 \mathrm{U} / \mathrm{mL}, H b A_{l c}$ glycated hemoglobin, $S D$ standard deviation

\section{Patient Data at Week 24}

Endpoint patient data at week 24 are summarized in Table 3. In the Asian population, the final dose of Gla-100 was $25.7 \pm 17.31 \mathrm{U}$ $(0.4 \pm 0.23 \mathrm{U} / \mathrm{kg} / \mathrm{day}), \quad \mathrm{HbA}_{1 \mathrm{c}}$ response was $-1.3 \pm 1.10 \%, \mathrm{HbA}_{1 \mathrm{c}}$ value was $7.5 \pm 0.96 \%$, and FPG was $6.1 \pm 1.52 \mathrm{mmol} / \mathrm{L}$. Amongst the Asian population, $31.9 \%$ achieved target glycemic control. In the Chinese subpopulation, the final dose of Gla-100 was $22.4 \pm 11.20 \mathrm{U}$ $(0.3 \pm 0.15 \mathrm{U} / \mathrm{kg} /$ day $), \quad \mathrm{HbA}_{1 \mathrm{c}}$ response was $-1.3 \pm 1.07 \%, \mathrm{HbA}_{1 \mathrm{c}}$ value was $7.2 \pm 0.82 \%$, and FPG was $6.0 \pm 1.23 \mathrm{mmol} / \mathrm{L}$. Amongst the Chinese subpopulation, $41.4 \%$ achieved target glycemic control.

\section{Primary Outcome}

Following multivariate regression analysis in the Asian population, BMI, FPG, duration of diabetes, and age at baseline were significantly associated with the dose of Gla-100 at week 24 (Fig. 1 and Table S2). Increases in BMI by $1 \mathrm{~kg} /$ $\mathrm{m}^{2}$ and FPG by $1 \mathrm{mmol} / \mathrm{L}$ at baseline were associated with an increase in Gla-100 dose at week 24 by $1.44 \mathrm{U}(P<0.0001)$ and $1.62 \mathrm{U}$ $(P<0.0001)$, respectively. An increase in the duration of diabetes by 1 year prior to the initiation of insulin was associated with a decrease in the dose of Gla-100 at week 24 by $0.23 \mathrm{U}$ $(P=0.0135)$. Similarly, an increase in age by 1 year at baseline was associated with a reduction in the dose of Gla-100 by $0.26 \mathrm{U}$ $(P=0.0001)$ at week 24 .

Figure 2 presents the nomogram with which to calculate the likely required dose of Gla-100 to achieve target glycemic control at week 24 , in Asian patients with T2DM. The dose of Gla-100 is calculated using all of the baseline covariates that were included in the multivariate analysis. The nomogram demonstrates that higher baseline BMI, FPG, and $\mathrm{HbA}_{1 \mathrm{c}}$, together with female gender, predict a higher dose requirement for Gla-100 to achieve target glycemic control at week 24. Longer duration of diabetes and older age predict a lower Gla-100 dose to achieve target glycemic control at week 24 .

Multivariate regression analysis in the subpopulation of patients from China demonstrated that BMI, FPG, age, and number of OADs at 
baseline were significantly associated with the dose of Gla-100 at week 24 (Fig. 1 and Table S2). As with the Asian population, increases in BMI by $1 \mathrm{~kg} / \mathrm{m}^{2}$ and FPG by $1 \mathrm{mmol} / \mathrm{L}$ at baseline were associated with an increase in the dose of Gla-100 at week 24 by $1.25 \mathrm{U}(P<0.0001)$ and $1.43 \mathrm{U}$ $(P<0.0001)$, respectively. Age and number of OADs, however, were negatively associated with the dose of Gla-100 at week 24. An increase in age by 1 year and treatment with one more type of OAD at baseline were associated with a decrease in the dose of Gla-100 at week 24 by $0.31 \mathrm{U}$ $(P<0.0001)$ and $4.78 \mathrm{U}(P=0.0032)$, respectively.

\section{Secondary Outcomes}

Multivariate analyses were also conducted to determine the impact of patient baseline covariates on treatment response and the dose of Gla-100 in units per kilogram per day (Fig. 3a for the Asian population and Fig. 3b for the Chinese subpopulation).
Multivariate analysis identified the following baseline predictors for $\mathrm{HbA}_{1 \mathrm{c}}$ at week 24: BMI $(P=0.0012)$ and $\mathrm{HbA}_{1 \mathrm{c}}(P<0.0001)$ in the Asian population and $\mathrm{HbA}_{1 \mathrm{c}}(P<0.0001)$ in the Chinese subpopulation. Baseline predictors for achievement of target glycemic control at week 24 were BMI $(P=0.0313)$, duration of diabetes $(P=0.0071)$, and $\mathrm{HbA}_{1 \mathrm{c}}(P<0.0001)$ in the Asian population and $\mathrm{HbA}_{1 \mathrm{c}}(P=0.0065)$ and FPG $(P=0.0382)$ in the Chinese subpopulation. Baseline predictors of $\mathrm{HbA}_{1 \mathrm{c}}$ response at week 24 were BMI $(P=0.0016)$ and $\mathrm{HbA}_{1 \mathrm{c}}$ $(P<0.0001)$ in the Asian population and $\mathrm{HbA}_{1 \mathrm{c}}$ $(P<0.0001)$ and age $(P=0.0218)$ in the Chinese subpopulation. Similarly, for FPG value at week 24, baseline predictors were identified as BMI $(P=0.0011)$ and FPG $(P<0.0001)$ in the Asian population and age $(P=0.0477)$, BMI $(P=0.0490)$, and FPG $(P=0.0022)$ in the Chinese subpopulation.

In terms of the dose of Gla-100 in units per kilogram per day at week 24 , identified baseline

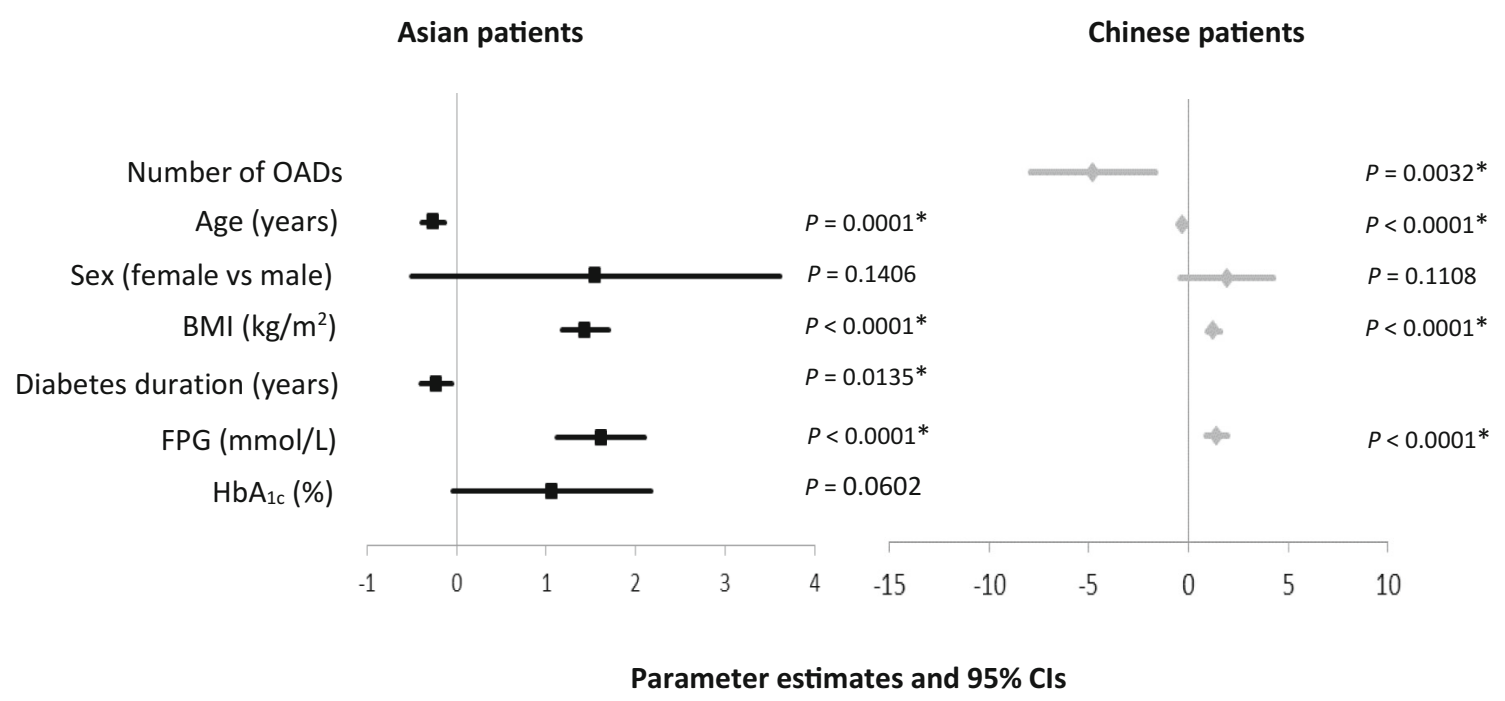

Fig. 1 Parameter estimates and 95\% confidence intervals for baseline predictors of Gla-100 dose at week 24 following multivariate regression analyses. Results are summarized for covariates included in the final model for each population. For the overall Asian population, the number of OADs prescribed at baseline did not satisfy the forward model entry selection criterion (from univariate to multivariate analysis) and hence was not included in the multivariate analysis. Similarly, for the Chinese patients, duration of diabetes and $\mathrm{HbA}_{1 \mathrm{c}}$ at baseline both failed to satisfy the entry criterion from univariate to multivariate analysis. Baseline covariates not included in the multivariate analyses for both populations have been left blank. $B M I$ body mass index, $C I$ confidence interval, FPG fasting plasma glucose, Gla-100 insulin glargine $100 \mathrm{U} / \mathrm{mL}$, $H b A_{1 c}$ glycated hemoglobin, $O A D s$ oral antidiabetic drugs. *Statistically significant 


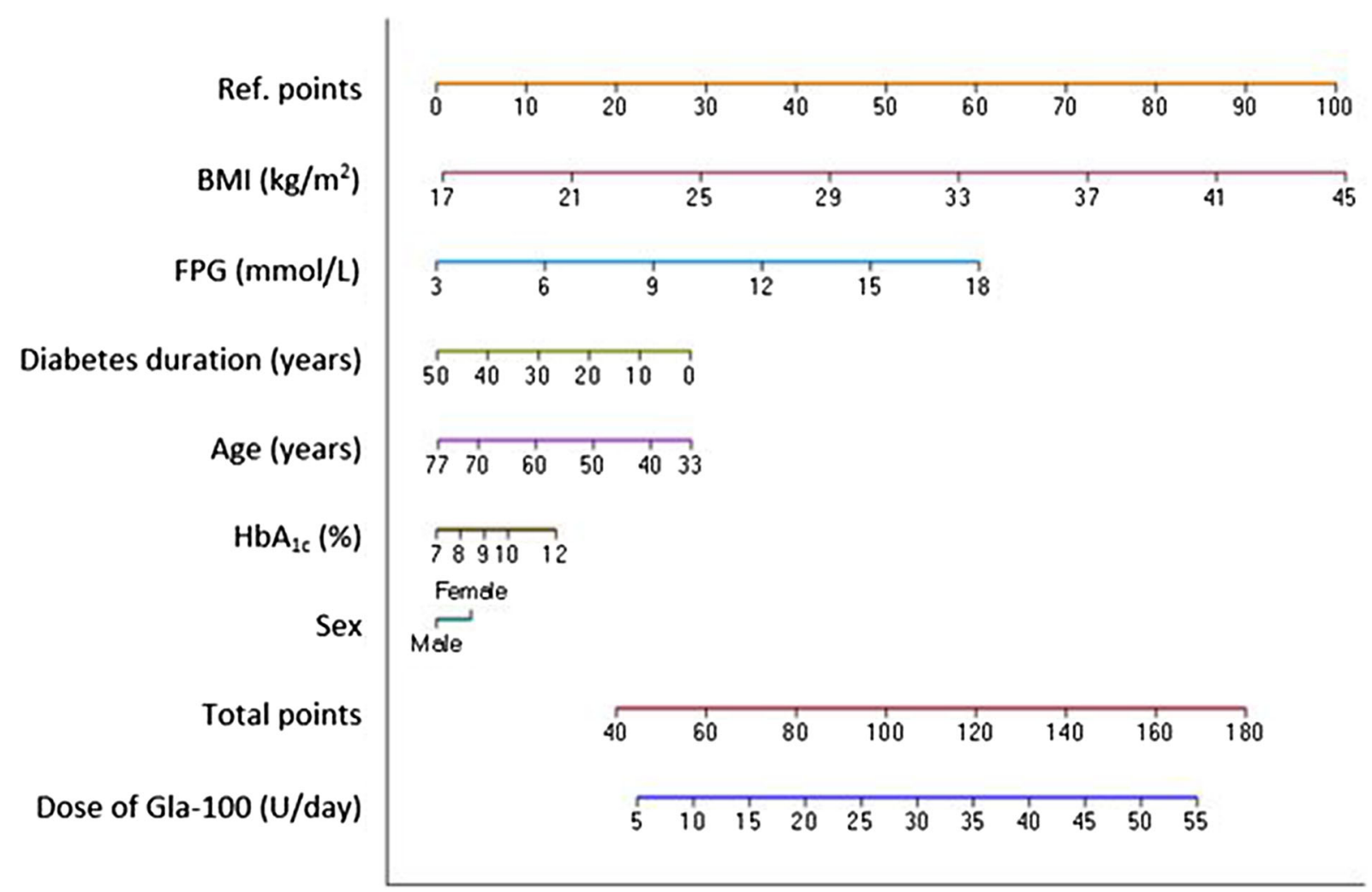

Fig. 2 Nomogram to predict the dose of Gla-100 likely to be required to achieve target glycemic control at week 24 . Note: choose the appropriate value of each of the baseline covariates, intercept perpendicularly the top horizontal line (Ref. points) and read the number. The sum of the ref. points, plotted on the "total points" line, corresponds to the prediction of the dose requirement for a patient at 24 weeks of treatment with Gla-100. For example, a

predictors were $\mathrm{HbA}_{1 \mathrm{c}} \quad(P=0.0160)$, age $(P<0.0001)$, sex $(P<0.0001)$, BMI $(P=0.0001)$, FPG $(P<0.0001)$, and duration of diabetes $(P=0.0057)$ in the Asian population. Identified predictors in the Chinese subpopulation were age $\quad(P=0.0013), \quad$ sex $\quad(P<0.0001), \quad$ BMI $(P=0.0244), \mathrm{HbA}_{1 \mathrm{c}}(P=0.0016)$, and number of OADs $(P=0.0022)$.

\section{DISCUSSION}

Using data from seven published individual TTT RCTs [1, 21-27], this pooled analysis aimed to determine the impact of patient baseline covariates on the dose of Gla-100 and its 60-year-old female patient who has had T2DM for a duration of 10 years (ref. points with BMI $25 \mathrm{~kg} / \mathrm{m}^{2}$, FPG $12 \mathrm{mmol} / \mathrm{L}$, and $\mathrm{HbA}_{1 \mathrm{c}} 10 \%$, approximately 113 total points) will likely require an insulin dose of $32 \mathrm{U} /$ day at week 24 to achieve target glycemic control. BMI body mass index, FPG fasting plasma glucose, Gla-100 insulin glargine $100 \mathrm{U} / \mathrm{mL}, H b A_{1 c}$ glycated hemoglobin

treatment response at week 24 in an overall population of Asian patients with T2DM and in a subpopulation of these patients from China. Identification of the predictors of dose and treatment response in the Chinese T2DM subpopulation was important to lay the foundation for future research specific to these individuals and to help guide local clinical practice. Results from the Chinese subpopulation were not intended as a comparison with the results obtained in the overall Asian population. For the purpose of this discussion, common baseline predictors for Asian patients and those from China have been elaborated on. Where deemed to be clinically relevant, predictors in the overall Asian population have also been 
(a)

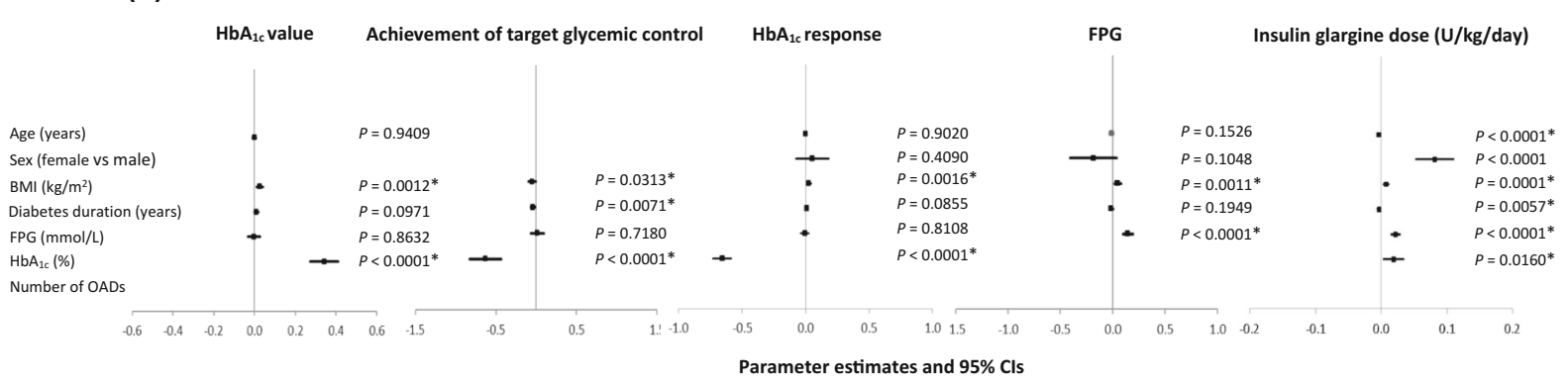

(b) Chinese subpopulation

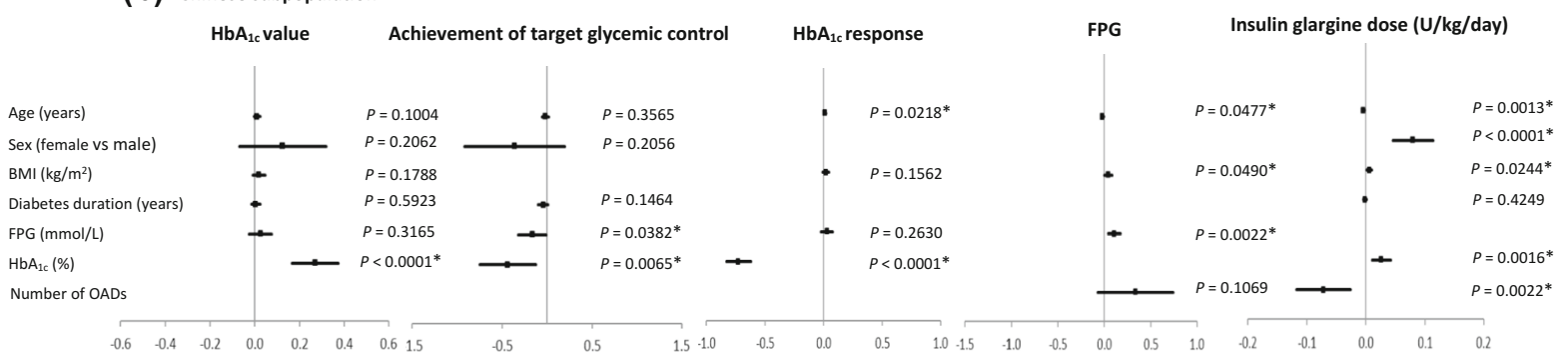

Parameter estimates and $95 \% \mathrm{Cls}$

Fig. 3 Parameter estimates and 95\% confidence intervals for baseline predictors of secondary outcomes at week 24 following multivariate analyses. Results are summarized for covariates included in the final model for each outcome/ population. Baseline covariates not included in the

highlighted. However, differences in observed results between the main population and subpopulation may have been affected by several factors, including the number of patients in each group. Further research and clinical validation of the results are therefore required to understand these differences and so are not discussed here.

The dose of Gla-100 at week 24 was negatively correlated with age and positively correlated with BMI and FPG in both the overall Asian population and the Chinese subpopulation. A post hoc analysis of the insulin glargine $\left(\right.$ Lantus $\left.^{\circledR}\right)$ vs. insulin detemir $\left(\right.$ Levemir $\left.{ }^{\circledR}\right)$ TreatTo-Target (L2T3) study, one of the RCTs included in this analysis, also demonstrated a similar relationship between final basal insulin dose (glargine or detemir) and the baseline characteristics of age, BMI, and FPG in patients with T2DM [28]. In the post hoc analysis, two multivariate analyses for the different outcomes in both populations have been left blank. $B M I$ body mass index, $C I$ confidence interval, $F P G$ fasting plasma glucose, $H b A_{I c}$ glycated hemoglobin, OADs oral antidiabetic drugs. * ${ }^{*}$ tatistically significant

stepwise regression analyses were performed, the first of which demonstrated that statistically significant predictors of a high final basal insulin dose, amongst others, were high FPG and younger age [28]. The second regression analysis, in which only physical characteristics that could be assessed "at the bedside" were included, demonstrated that high BMI, high FPG, and younger age were predictors of a high final basal insulin dose [28]. The study acknowledged that rough estimations of the final basal insulin dose can be made when taking into account a patient's BMI and age [28].

Similar to the primary outcome, the dose of Gla-100 expressed in units per kilogram per day at week 24 was also shown to be negatively correlated with age and positively correlated with BMI and FPG in the Asian population. Post hoc analysis of the L2T3 study also reported a positive correlation between BMI and the final 
basal insulin dose expressed in terms of weight; however, data supporting this outcome were not shown [28].

These results suggest that a patient with higher FPG at baseline requires a higher dose of Gla-100 to reduce their FPG level, which is in accordance with clinical expectations. The relationship between BMI and dose of Gla-100 has a physiological basis. Higher BMI has been shown to be associated with decreased insulin sensitivity $[29,30]$, and a higher dose may be required in patients with higher BMI in order to stimulate insulin absorption and decrease glycemic levels. Indeed, titration based on a patient's weight (an important factor in determining BMI) is thought to have a stronger physiological basis than glucose level or dosebased regimens, especially given the relationship between body weight and insulin sensitivity [31]. As mentioned above, the post hoc analysis of the L2T3 study also demonstrated a negative correlation between age and the final dose of basal insulin; however, the authors of the study acknowledged that there are no straightforward explanations for this finding [28]. The authors of the current study hypothesize that younger patients, who are in need of stricter glycemic control $[4,5,32]$ and who have been shown to be at a lower risk of hypoglycemia [33-35], are likely to have had their doses of Gla-100 titrated to a greater extent than older patients, leading to the observed relationship between the dose of Gla-100 at week 24 and age.

The above analyses indicate that a higher dose of basal insulin is likely to be required in younger patients or patients with higher baseline BMI or FPG, and physicians must therefore titrate the insulin doses of these patients to an adequate level to achieve glycemic targets. The opposite must also be taken into consideration in older patients or patients with lower baseline BMI or FPG, for whom lower doses of basal insulin may be required to achieve glycemic targets. In these patients, a more cautious approach to the titration of basal insulin is warranted.

The nomogram developed in this study can help clinicians to determine the dose of Gla-100 likely to be required by a patient to achieve target glycemic control at week 24, thereby providing guidance for the appropriate titration of Gla-100 according to a TTT algorithm. Further details on interpretation of the nomogram are included in Fig. 2. The nomogram developed in this study is a representation of the results of the multivariate analysis of the primary outcome as a whole and includes all the patient baseline covariates included in the analysis, regardless of whether they were significant or not. Therefore, although not significant, baseline $\mathrm{HbA}_{1 \mathrm{c}}$, duration of diabetes, and gender have also been included in the nomogram. With regard to baseline $\mathrm{HbA}_{1 \mathrm{c}}$, post hoc analysis of the L2T3 study demonstrated similar results, with multivariate analyses demonstrating that the baseline level of $\mathrm{HbA}_{1 \mathrm{c}}$ was not a significant determinant of the final basal insulin dose [28]. It should be noted that the dose of Gla-100 predicted by the nomogram developed in this study is only an estimation of the required dose to achieve target glycemic control at week 24 and does not enable calculation of the initial therapeutic dose of Gla-100. In general, Asian country-specific guidelines recommend a dose of $0.1-0.3 \mathrm{U} / \mathrm{kg} /$ day for the initiation of basal insulin [6, 36-38].

A formula for determining the total optimal daily dose of Gla-100 at 24 weeks, based on both the optimal starting and incremental doses, has been previously developed using data from a 24-week observational study of Japanese patients with T2DM [39]. The objective and parameters included in the formula differ, however, from those of the nomogram developed in the present analysis; furthermore, the formula was derived from observational data from a single study wherein physicians were free to titrate according to their clinical practice [39]. The nomogram in the current study was developed using data from seven RCTs, each with its own starting doses and TTT algorithms based on FPG [1, 21-27]. The patient populations used in both analyses also differ; the aforementioned formula was derived from a population that achieved target $\mathrm{HbA}_{1 \mathrm{c}}$ [39], whereas the nomogram in the current study was constructed using data from the FAS populations of the individual TTT RCTs. Hence, direct comparisons between the formula and the 
nomogram developed in the current study cannot be made.

The multivariate analyses demonstrated a statistically significant positive correlation between $\mathrm{HbA}_{1 \mathrm{c}}$ value at baseline and $\mathrm{HbA}_{1 \mathrm{c}}$ value at week 24 in both the Asian population and the Chinese subpopulation. In a study by Fujita et al. [40], multiple linear regression analysis was conducted to determine the characteristics influencing the effectiveness of Gla100 treatment in insulin-naïve T2DM Japanese patients with suboptimal glycemic control on OADs. $\mathrm{HbA}_{1 \mathrm{c}}$ at baseline was shown to be a statistically significant predictor of $\mathrm{HbA}_{1 \mathrm{c}}$ at week $24(P=0.006)$, with an increase at baseline predicting a higher endpoint $\mathrm{HbA}_{1 \mathrm{c}}$ value [40].

In the present study, multivariate analyses also demonstrated a negative association between achieving target glycemic control $\left(\mathrm{HbA}_{1 \mathrm{c}}<7 \%\right)$ at week 24 and baseline $\mathrm{HbA}_{1 \mathrm{c}}$ in the Asian population and Chinese subpopulation. The negative association between achieving target glycemic control and baseline $\mathrm{HbA}_{1 \mathrm{c}}$ has been reported in several studies aimed at identifying the characteristics associated with glycemic response to newly initiated insulin therapy in both Asian and Western populations with T2DM [41-43]. In particular, a subjectlevel meta-analysis of 12 RCTs that used Gla100 in a TTT titration regimen in patients with T2DM, including some of the RCTs involved in the current pooled analysis, demonstrated that baseline $\mathrm{HbA}_{1 \mathrm{c}}$ was negatively associated with achieving $\mathrm{HbA}_{1 \mathrm{c}} \leq 7.0 \%$ [43]. In an observational study of newly initiated insulin therapy in patients with T2DM by Nichols et al. [42], multivariate analyses demonstrated that $\mathrm{HbA}_{1 \mathrm{c}}$ prior to insulin initiation was the dominant factor in predicting treatment goal attainment $\left(\mathrm{HbA}_{1 \mathrm{c}}<7 \%\right)$, and that a $1 \%$ increase in $\mathrm{HbA}_{1 \mathrm{c}}$ prior to insulin initiation reduced the probability of attaining target glycemic control by $26 \%$ [42]. In the current analysis, a 1\% increase in $\mathrm{HbA}_{1 \mathrm{c}}$ prior to initiation of Gla-100 reduced the probability of attaining target glycemic control by $63.1 \%$ in the Asian population and by $43.7 \%$ in the Chinese population.

In addition to baseline $\mathrm{HbA}_{1 \mathrm{c}}$, duration of diabetes was also negatively associated with achieving target glycemic control at week 24 in Asian patients. The aforementioned studies by Fujita et al. [40] and Nichols et al. [42] have also reported that a longer duration of diabetes is associated with a lower likelihood of achieving target glycemic control.

Validating the above, multivariate analyses conducted in this study demonstrated a negative correlation between $\mathrm{HbA}_{1 \mathrm{c}}$ response (reduction from baseline to week 24) and baseline $\mathrm{HbA}_{1 \mathrm{c}}$ in both the Asian population and the Chinese subpopulation. Predictors of change in $\mathrm{HbA}_{1 \mathrm{c}}$ were also investigated using data from the large, 24-week, observational $\mathrm{A}_{1}$ chieve study, which involved patients with T2DM initiating insulin therapy. In both predictor and explanatory analyses, $\mathrm{HbA}_{1 \mathrm{c}}$ level at baseline was negatively associated with change in $\mathrm{HbA}_{1 \mathrm{c}}$ from baseline to the endpoint [44].

This above evidence indicates that baseline $\mathrm{HbA}_{1 \mathrm{c}}$ is an important factor in determining the level of glycemic control attained. In the study by Nichols et al. [42], $\mathrm{HbA}_{1 \mathrm{c}}$ prior to starting insulin therapy accounted for $95 \%$ of the discriminatory ability to predict the probability of attaining target glycemic control and $96 \%$ of the explainable variance in $\mathrm{HbA}_{1 \mathrm{c}}$ change. These observations are expected, since a patient with $\mathrm{HbA}_{1 \mathrm{c}}$ closer to $7 \%$ should achieve target glycemic control more easily following treatment initiation with insulin. This, however, does not eliminate the fact that in several studies, patients with T2DM who achieved target glycemic control also had a greater reduction in $\mathrm{HbA}_{1 \mathrm{c}}$ after initiating insulin therapy, despite a lower mean $\mathrm{HbA}_{1 \mathrm{c}}$ at baseline [41, 42].

Baseline predictors for FPG value at week 24 were also investigated in our study. To the best of our knowledge, other studies demonstrating similar results have not been conducted, and hence these novel findings require further investigation. Common baseline predictors in the Asian population and Chinese subpopulation were BMI and FPG, both being positively associated with FPG at week 24 .

There are several limitations to this study. No methods were used to assess risk of bias within the individual studies or across the included studies. Additionally, selection of the patient baseline covariates included in the 
analyses was limited by the available data in the individual RCTs. This pooled analysis, however, provides results from a larger group of Asian and Chinese patients compared with each of the individual seven studies. Additionally, this study performed separate analyses for patients from Asia and for the subpopulation of patients from China, in order to explore the relationship between patient characteristics and outcomes in each of the populations. Understanding the reasons behind differences between the populations' results requires further research involving larger patient populations and validation of these results. The nomogram developed in this study requires further independent clinical validation. Future studies could also look to develop and assess nomograms based on a wider range of patient characteristics, such as the presence of diabetes complications or comorbidities. Additional research involving a larger pool of baseline covariates, including threshold homeostasis model assessment (HOMA) of insulin resistance levels, HOMA of $\beta$-cell function levels, and C-peptide may provide more insight into the impact of insulin resistance and secretion capacity on the dose of Gla-100 and treatment response. Investigating different patient profiles could also explain and establish more in-depth and clinically relevant correlations, such as those associated with the risk of occurrence of hypoglycemia.

\section{CONCLUSIONS}

This pooled analysis identified the main baseline predictors of important therapeutic parameters, such as the dose of Gla-100 and treatment response at week 24 in an overall population of Asian patients with T2DM and a subpopulation of these patients from China. In both populations, results indicated that a higher dose of Gla-100 is likely to be required in younger patients, or in patients with higher baseline BMI or FPG, and that physicians must titrate the doses of basal insulin for these patients to an appropriate level in order for them to achieve glycemic targets. Aiding appropriate titration, the nomogram developed in this study enables calculation of the dose of
Gla-100 likely to be required by Asian patients with T2DM to achieve $\mathrm{HbA}_{1 \mathrm{c}}<7 \%$ at week 24 . In both the study populations, higher baseline $\mathrm{HbA}_{1 \mathrm{c}}$ was associated with lower reduction in $\mathrm{HbA}_{1 \mathrm{c}}$ from baseline, higher $\mathrm{HbA}_{1 \mathrm{c}}$ at week 24, and a lower chance of achieving target glycemic control at week 24; this indicates that baseline $\mathrm{HbA}_{1 \mathrm{c}}$ is an important factor in determining the level of glycemic control attained following treatment with Gla-100. The achievement of target glycemic control at week 24 was also negatively associated with duration of diabetes in the Asian population, demonstrating the importance of timely initiation of basal insulin.

\section{ACKNOWLEDGEMENTS}

Funding. This pooled analysis and the trials involved in it were sponsored by Sanofi. Sanofi was responsible for the design of the study, collecting and managing data, and performing all statistical analyses. Article processing charges were also funded by Sanofi.

Editorial Assistance. Editorial assistance in the preparation of this article was provided by Priya Shreedhar of MediTech Media Asia Pacific and was funded by Sanofi.

Authorship. All named authors meet the International Committee of Medical Journal Editors (ICMJE) criteria for authorship for this manuscript, take responsibility for the integrity of the work as a whole, and have given final approval for the version to be published. All authors had full access to all of the data in this study and take complete responsibility for the integrity of the data and accuracy of the data analysis.

Disclosures. Tianwei Gu, Ting Hong, Pengzi Zhang, Sunyinyan Tang, Yan Bi, and Dalong Zhu declare that they have no conflict of interest. Hai $\mathrm{Lu}$ is an employee of Sanofi. Dongwei $\mathrm{Ma}$ is also an employee of Sanofi. Lichuang Men was an employee of Sanofi at the time of the study. 
Compliance with Ethics Guidelines. This article is based on previously conducted studies and does not involve any new studies of human or animal subjects performed by any of the authors.

Data Availability. All data generated or analyzed during this study are included in this published article/as supplementary information files.

Open Access. This article is distributed under the terms of the Creative Commons Attribution-NonCommercial 4.0 International License (http://creativecommons.org/licenses/ by-nc/4.0/), which permits any noncommercial use, distribution, and reproduction in any medium, provided you give appropriate credit to the original author(s) and the source, provide a link to the Creative Commons license, and indicate if changes were made.

\section{REFERENCES}

1. Riddle MC, Rosenstock J, Gerich J. The treat-totarget trial: randomized addition of glargine or human NPH insulin to oral therapy of type 2 diabetic patients. Diabetes Care. 2003;26:3080-6.

2. Turner RC, Cull CA, Frighi V, Holman RR. Glycemic control with diet, sulfonylurea, metformin, or insulin in patients with type 2 diabetes mellitus: progressive requirement for multiple therapies (UKPDS 49). JAMA. 1999;281:2005-12.

3. American Diabetes Association. Pharmacologic approaches to glycemic treatment: standards of medical care in diabetes-2018. Diabetes Care. 2018;41(Suppl 1):S73-85.

4. Garber AJ, Abrahamson MJ, Barzilay JI, et al. Consensus statement by the American Association of Clinical Endocrinologists and American College of Endocrinology on the comprehensive type 2 diabetes management algorithm-2018 executive summary. Endocr Pract. 2018;24:91-120.

5. International Diabetes Federation. Global guideline for type 2 diabetes. 2012. https://www.idf.org/ouractivities/advocacy-awareness/resources-and-tools/ 79:global-guideline-for-type-2-diabetes.html. Accessed Jan 2018.
6. Ji L, Lu J, Zhu D, et al. Chinese expert recommendations on basal insulin treatment in adult type 2 diabetes mellitus. Chin J Diabetes. 2017;25:2-9.

7. Ji L, Tsai ST, Lin J, Bhambani S. National variations in comorbidities, glycosylated hemoglobin reduction, and insulin dosage in Asian patients with type 2 diabetes: the FINE-Asia registry. Diabetes Ther. 2015;6:519-30.

8. Ji L, Zhang P, Weng J, et al. Observational Registry of Basal Insulin Treatment (ORBIT) in patients with type 2 diabetes uncontrolled by oral hypoglycemic agents in China-study design and baseline characteristics. Diabetes Technol Ther. 2015;17:735-44.

9. Tsai ST, Pathan F, Ji L, et al. First insulinization with basal insulin in patients with type 2 diabetes in a real-world setting in Asia. J Diabetes. 2011;3:208-16.

10. Lim U, Ernst T, Buchthal SD, et al. Asian women have greater abdominal and visceral adiposity than Caucasian women with similar body mass index. Nutr Diabetes. 2011;1:e6.

11. Menke A, Casagrande S, Geiss L, Cowie CC. Prevalence of and trends in diabetes among adults in the United States, 1988-2012. JAMA. 2015;314:1021-9.

12. Wang J, Thornton JC, Russell M, et al. Asians have lower body mass index (BMI) but higher percent body fat than do whites: comparisons of anthropometric measurements. Am J Clin Nutr. 1994;60:23-8.

13. Pan CY, Sinnassamy P, Chung KD, Kim KW. Insulin glargine versus NPH insulin therapy in Asian type 2 diabetes patients. Diabetes Res Clin Pract. 2007;76:111-8.

14. Ji L, Zhang P, Zhu D, et al. Observational Registry of Basal Insulin Treatment (ORBIT) in patients with type 2 diabetes uncontrolled with oral antihyperglycaemic drugs: real-life use of basal insulin in China. Diabetes Obes Metab. 2017;19:822-30.

15. Scheen AJ, Schmitt H, Jiang HH, Ivanyi T. Individualizing treatment of type 2 diabetes by targeting postprandial or fasting hyperglycaemia: response to a basal vs a premixed insulin regimen by $\mathrm{HbA}_{1 \mathrm{c}}$ quartiles and ethnicity. Diabetes Metab. $2015 ; 41: 216-22$.

16. Sheu WH, Brunell SC, Blase E. Efficacy and tolerability of exenatide twice daily and exenatide once weekly in Asian versus White patients with type 2 diabetes mellitus: a pooled analysis. Diabetes Res Clin Pract. 2016;114:160-72.

17. Yoon YS, Choi HS, Kim JK, Kim YI, Oh SW. Differences in the associations of anthropometric 
measures with insulin resistance and type 2 diabetes mellitus between Korean and US populations: comparisons of representative nationwide sample data. Obes Res Clin Pract. 2016;10:642-51.

18. Chiu M, Austin PC, Manuel DG, Shah BR, Tu JV. Deriving ethnic-specific BMI cutoff points for assessing diabetes risk. Diabetes Care. 2011;34:1741-8.

19. Ma RC, Chan JCN. Type 2 diabetes in East Asians: similarities and differences with populations in Europe and the United States. Ann N Y Acad Sci. 2013;1281:64-91.

20. Chan JCN, Bunnag P, Chan SP, et al. Glycaemic responses in Asian and non-Asian people with type 2 diabetes initiating insulin glargine 100 units/mL: a patient-level pooled analysis of 16 randomised controlled trials. Diabetes Res Clin Pract. 2018;135:199-205.

21. Aschner P, Sethi B, Gomez-Peralta F, et al. Insulin glargine compared with premixed insulin for management of insulin-naive type 2 diabetes patients uncontrolled on oral antidiabetic drugs: the openlabel, randomized GALAPAGOS study. J Diabetes Complications. 2015;29:838-45.

22. ATLAS Study Group. Titration of insulin glargine in patients with type 2 diabetes mellitus in Asia: physician- versus patient-led? Rationale of the Asian Treat to Target Lantus Study (ATLAS). Diabetes Technol Ther. 2011;13:67-72.

23. Fritsche A, Schweitzer MA, Haring HU. Glimepiride combined with morning insulin glargine, bedtime neutral protamine hagedorn insulin, or bedtime insulin glargine in patients with type 2 diabetes. Ann Intern Med. 2003;138:952-9.

24. Garg SK, Admane K, Freemantle N, et al. Patient-led versus physician-led titration of insulin glargine in patients with uncontrolled type 2 diabetes: a randomized multinational ATLAS study. Endocr Pract. 2015;21:143-57.

25. Gerstein HC, Yale JF, Harris SB, et al. A randomized trial of adding insulin glargine vs. avoidance of insulin in people with type 2 diabetes on either no oral glucose-lowering agents or submaximal doses of metformin and/or sulphonylureas. The Canadian INSIGHT (Implementing New Strategies with Insulin Glargine for Hyperglycaemia Treatment) Study. Diabet Med. 2006;23:736-42.

26. Swinnen SG, Snoek FJ, Dain MP, et al. Rationale, design, and baseline data of the insulin glargine (Lantus) versus insulin detemir (Levemir) Treat-ToTarget (L2T3) study: a multinational, randomized noninferiority trial of basal insulin initiation in type 2 diabetes. Diabetes Technol Ther. 2009;11:739-43.

27. Yki-Jarvinen H, Juurinen L, Alvarsson M, et al. Initiate Insulin by Aggressive Titration and Education (INITIATE): a randomized study to compare initiation of insulin combination therapy in type 2 diabetic patients individually and in groups. Diabetes Care. 2007;30:1364-9.

28. Simon AC, Bolli GB, Dain MP, Wang E, Holleman F. Physical characteristics that predict final basal insulin dose in type 2 diabetes mellitus, with a special focus on BMI. Nutr Metab Cardiovasc Dis. 2014;24:1354-9.

29. Chung JO, Cho DH, Chung DJ, Chung MY. Associations among body mass index, insulin resistance, and pancreatic beta-cell function in Korean patients with new-onset type 2 diabetes. Korean J Intern Med. 2012;27:66-71.

30. Walton C, Godsland IF, Proudler AJ, Felton CV, Wynn V. Effect of body mass index and fat distribution on insulin sensitivity, secretion, and clearance in nonobese healthy men. J Clin Endocrinol Metab. 1992;75:170-5.

31. Li X, Du T, Li W, et al. Efficacy and safety of weightbased insulin glargine dose titration regimen compared with glucose level- and current dose-based regimens in hospitalized patients with type 2 diabetes: a randomized, controlled study. Clin Ther. 2014;36:1269-75.

32. American Diabetes Association. Glycemic targets: standards of medical care in diabetes-2018. Diabetes Care. 2018;41(Suppl 1):S55-64.

33. Bramlage $\mathrm{P}$, Gitt $\mathrm{AK}$, Binz $\mathrm{C}$, et al. Oral antidiabetic treatment in type- 2 diabetes in the elderly: balancing the need for glucose control and the risk of hypoglycemia. Cardiovasc Diabetol. 2012;11:122.

34. Giorda CB, Ozzello A, Gentile S, et al. Incidence and correlates of hypoglycemia in type 2 diabetes. The Hypos-1 Study. J Diabetes Metab. 2014;5:344.

35. Henderson JN, Allen KV, Deary IJ, Frier BM. Hypoglycaemia in insulin-treated type 2 diabetes: frequency, symptoms and impaired awareness. Diabet Med. 2003;20:1016-21.

36. Department of Health of the Government of the Hong Kong Special Administrative Region. Hong Kong reference framework for diabetes care for adults in primary care settings. 2017. http://www. pco.gov.hk/english/resource/files/RF_DM_full.pdf. Accessed Jan 2018.

37. Ministry of Health Malaysia. Practical guide to insulin therapy in type 2 diabetes mellitus. 2011. http:// 
www.mems.my/file_dir/3308086634dc0e0f9e1c72. pdf. Accessed Jan 2018.

38. Philippine Society of Endocrinology Diabetes and Metabolism. Philippine practice guidelines on the diagnosis and management of diabetes mellitus. 2014. http://endo-society.org.ph/v5/wp-content/ uploads/2013/06/Diabetes-United-for-Diabetes-Phil. pdf. Accessed Jan 2018.

39. Kadowaki T, Ohtani T, Naito Y, Odawara M. Potential formula for the calculation of starting and incremental insulin glargine doses: ALOHA subanalysis. PLoS One. 2012;7:e41358.

40. Fujita N, Tsujii S, Kuwata $\mathrm{H}$, et al. Predictor variables and an equation for estimating HbA1c attainable by initiation of basal supported oral therapy. J Diabetes Investig. 2012;3:164-9.

41. Lin SD, Tsai ST, Tu ST, et al. Glycosylated hemoglobin level and number of oral antidiabetic drugs predict whether or not glycemic target is achieved in insulin-requiring type 2 diabetes. Prim Care Diabetes. 2015;9:135-41.

42. Nichols GA, Kimes TM, Harp JB, Kou TD, Brodovicz KG. Glycemic response and attainment of A1C goals following newly initiated insulin therapy for type 2 diabetes. Diabetes Care. 2012;35:495-7.

43. Riddle MC, Vlajnic A, Zhou R, Rosenstock J. Baseline $\mathrm{HbA} 1 \mathrm{c}$ predicts attainment of $7.0 \% \mathrm{HbA} 1 \mathrm{c}$ target with structured titration of insulin glargine in type 2 diabetes: a patient-level analysis of 12 studies. Diabetes Obes Metab. 2013;15:819-25.

44. Home PD, Shen C, Hasan MI, et al. Predictive and explanatory factors of change in $\mathrm{HbA}_{1 \mathrm{c}}$ in a 24-week observational study of 66,726 people with type 2 diabetes starting insulin analogs. Diabetes Care. 2014;37:1237-45. 